\title{
Intentional GNSS Interference Detection and Characterization Algo- rithm Using AGC and Adaptive IIR Notch Filter
}

\author{
Jeong Hwan Yang*, Chang Ho Kang**, Sun Young Kim*** and Chan Gook Park****
}

School of Mechanical and Aerospace Engineering/Automation and System Research Institute/

The Institute of Advanced Aerospace Technology, Seoul National University, Seoul 151-742, Korea

\begin{abstract}
A Ground Based Augmentation System (GBAS) is an enabling technology for an aircraft's precision approach based on a Global Navigation Satellite System (GNSS). However, GBAS is vulnerable to interference, so effective GNSS interference detection and mitigation methods need to be employed. In this paper, an intentional GNSS interference detection and characterization algorithm is proposed. The algorithm uses Automatic Gain Control (AGC) gain and adaptive notch filter parameters to classify types of incoming interference and to characterize them. The AGC gain and adaptive lattice IIR notch filter parameter values in GNSS receivers are examined according to interference types and power levels. Based on those data, the interference detection and characterization algorithm is developed and Monte Carlo simulations are carried out for performance analysis of the proposed method. Here, the proposed algorithm is used to detect and characterize single-tone continuous wave interference, swept continuous wave interference, and band-limited white Gaussian noise. The algorithm can be used for GNSS interference monitoring in an excessive Radio Frequency Interference environment which causes loss of receiver tracking. This interference detection and characterization algorithm will be used to enhance the interference mitigation algorithm.
\end{abstract}

Key words: GBAS, GNSS interference detection, GNSS interference monitoring

\section{Introduction}

The GNSS signal power level at a receiver is extremely low. Therefore, radio frequency interference (RFI) is a serious concern, particularly for GNSS-based safetycritical systems such as the Ground Based Augmentation System (GBAS). Non-Fed CAT I GBAS was approved by FAA in September 2009. However, its operational stability test failed due to a privacy jammer's intentional interference. This incident showed that the GBAS was not designed robustly against intentional jamming. After upgrading GBAS software and implementing a physical configuration change and law enforcement against jamming, the system became more stable but it is still vulnerable to excessive radio frequency interference [1]. GBAS equipment's RFI requirement in ICAO ANEX 10 states that GBAS receivers shall not output misleading information in the presence of interference levels exceeding the specified RFI mask, which are $-120.5 \mathrm{dBm}$ for inband continuous wave interference and $-110.5 \mathrm{dBm} / \mathrm{MHz}$ for broadband interference [2]. These requirements ensure safe flight operation by making the receivers provide navigation information only in a normal radio frequency environment. However, for safe flight operation, there should be a system which provides situational awareness even in an excessive radio frequency interference environment. This is especially true in Korea. In the past two years, South Korea has experienced intentional GPS jamming several times. By investigation, it was found that the incident was due to North Korea's intentional jamming and the types of jamming signals
This is an Open Access article distributed under the terms of the Creative Commons Attribution Non-Commercial License (http://creativecommons.org/licenses/by$\mathrm{nc} / 3.0 /$ which permits unrestricted non-commercial use, distribution, and reproduction in any medium, provided the original work is properly cited.

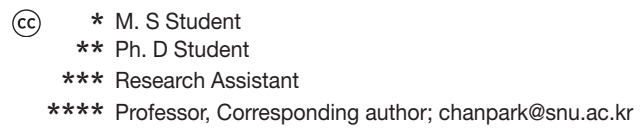


were single-tone continuous wave on L1, and swept continuous wave on L2 and L5 frequency bands [3]. GPS jammers in place near the Demilitarized Zone (DMZ) could easily disrupt GPS signals in Incheon international airport from 50 kilometers away. Therefore, providing timely alert by detection and characterization of GNSS RFI, and eliminating the possible cause in a timely manner are important to ensure safe flight operation based on the GBAS.

There are various methods to detect GNSS interference. They are mainly classified as the pre-correlation detection method and the post-correlation detection method depending on where the algorithms are applied. Antenna, AGC and ADC have been used to detect and characterize the RFI in pre-correlation techniques [4-7], while receiver observables like $\mathrm{C} / \mathrm{No}$, phase distortion, and pseudo-range step measurements are used for post-correlation techniques [8-11]. For most civil applications, post correlation detection methods using receiver observables have been preferred because they do not require additional hardware modification. However, post-correlation methods can be used only when a receiver is tracking the GNSS signal, so the methods are not adequate for characterization of intentional interference whose power is above a civil receiver's tracking threshold. Also, post-correlation methods require more time to detect interference than pre-correlation methods because $\mathrm{C} /$ No estimation requires an averaging time of approximately 1 second to achieve unbiased estimation results [12]. To overcome these limitations, this paper is focused on the pre-correlation method using AGC gain and adaptive lattice IIR filter parameters, which can be used for intentional interference which causes loss of tracking. The algorithm is developed to detect and characterize single-tone continuous wave interference, swept continuous wave interference and band-limited white noise, which are the most common intentional interferences [4]. For the verification and performance evaluation of the proposed algorithm, GPS signal data in various interference scenarios are generated using a GPS and interference simulator. The performance of the algorithm was analyzed by Monte Carlo simulation and the simulation results showed that the algorithm is capable of detection and characterization of the specified intentional interferences.

This paper is organized as follows: the GPS signal and interference simulator are described in section 2. In section 3, the AGC model and adaptive lattice IIR notch filter parameters are explained and section 4 describes the proposed interference detection and characterization algorithm. The algorithm's performance is evaluated in section 5 and finally section 6 concludes this paper.

\section{GNSS Signal and Interference Simulator}

GNSS signals are modeled differently depending on the signal modulation scheme. However, the proposed interference detection and characterization algorithm utilizes the signals before the correlation process, so considering every different modulation scheme is not necessary for the development of the algorithm. In this paper, a MATLAB SIMULINK-based GPS L1 signal and interference simulator is used to assess AGC gains and generate IF signal data in various interference scenarios [13]. The simulator generates a GPS L1 C/A signal, which is modeled as Eq. (1).

$$
r(t)=A \times D(t-\tau) c(t-\tau) \cos \left(2 \pi\left(f_{L 1}+f_{d}\right) t+\theta\right)+n(t)+i(t)
$$

where $A$ is IF signal amplitude, $D(t)$ is navigation data, $C(t)$ is $\mathrm{C} / \mathrm{A}$ code, $\tau$ is code phase, $f_{d}$ is Doppler frequency shift, $\theta$ is carrier phase, $n(t)$ is noise, and $i(t)$ is interference.

The Additive White Gaussian Noise (AWGN) is modeled by a variance of $\sigma_{n}^{2}=\frac{N_{0} f_{s}}{2}$, where $\frac{N_{0}}{2}$ is the power spectral density of the noise and $f_{2}$ is the sampling frequency [14]. In addition, single-tone and swept continuous wave interferences are modeled as:

$$
\begin{aligned}
& i_{c w i}(t)=\sqrt{2 P_{i}} \cos \left(2 \pi f_{i} t+\phi_{i}\right) \\
& i_{\text {swept cwi }}(t)=\sqrt{2 P_{i}} \cos \left(2 \pi f_{i}(t) t+\phi_{i}\right)
\end{aligned}
$$

where $P$ is signal power, $f_{i}$ is interference frequency and is phase.

The band-limited white noise is generated using the bandlimited white noise block and band pass filter block. Using the simulator, users can select the type of interference and adjust power and duration. The simulator generates the GPS signal and combines it with noise and interference, then the signal goes through band pass filter, AGC, ADC blocks and it is saved to a file in binary format. Also, AGC gain values are saved to a file which will be used as a database for AGC characterization.

\section{AGC and Adaptive Lattice IIR Notch Filter Parameters for Interference Detection}

\subsection{Automatic Gain Control}

\subsubsection{AGC model}

The Automatic Gain Control (AGC) in a Global Navigation Satellite System receiver is used to adjust the power level of the IF signal at the input to the Analog-to-Digital Converter 
(ADC) to minimize the quantization loss [15]. The AGC level as an interference assessment tool has been investigated before [16]. In GNSS receivers, where the signal power is below the thermal noise floor, the AGC is driven by the ambient noise or interference rather than the signal power. The interfering signal is a main source that changes the AGC gain. Therefore, AGC can be a valuable tool for assessing the interference $[2,17]$. AGC is sensitive to both wide band noise and continuous wave interferences. In addition, its gain responds differently depending on the type of interferences $[2,17,18]$. Therefore, if a receiver's AGC is characterized against various interferences in advance and an incoming interference type is known, interference power can be estimated from the receiver's AGC gain. In this paper, the simulator's AGC gain response was examined according to different types of interference and the data were used for power estimation of interference sources.

As previously mentioned, without intentional jamming, the thermal noise with Gaussian distribution dominates the received signal power strength. The RMS amplitude of the thermal noise plus jamming noise must remain essentially constant at the input of the ADC. When RF interference occurs, the action of the AGC is to rapidly reduce the gain in order to maintain the original RMS level at the input of the ADC. The analog gain control voltage has an effect on the gain of the AGC as follows:

$$
G_{A}=\alpha e^{\beta V_{A G C}}
$$

where: $V_{A G C}$ is the AGC control voltage, $\alpha$ is the AGC gain coefficient, and $\beta$ is the AGC control voltage coefficient.

The two coefficients are different depending on manufacturers, and even the same front-end models have slightly different characteristics [17]. However, if an AGC has a preplanned design and it is characterized for a receiver in interference environments, the receiver's AGC gain values can be used for interference detection [19]. For algorithm development, the GNSS signal and interference simulator is adapted an exponential AGC model which is controlled by a digital feedback loop and 8 bit ADC. AGC gain values in different interference scenarios are saved for characterization.

\subsubsection{AGC Characterization}

This section presents an analysis of the interference effects on AGC gain according to the types of interference at different power levels. Interference types used for AGC characterization include single-tone continuous wave interference at a GPS center frequency, swept continuous wave interference with $500 \mathrm{MHz} / \mathrm{sec}$ in a $4 \mathrm{MHz}$ band width, and band limited white noise in a $4 \mathrm{MHz}$ band width. The received GPS signal power level was set to $-155 \mathrm{dBW}$, and power spectral density of the Additive White Gaussian Noise was assumed to be $-204 \mathrm{dBW} / \mathrm{Hz}$. The GNSS signal and interference simulator generates signals by increasing interference power and AGC gain values are logged in a file. The AGC Characterization results are shown in Fig 1. AGC gain decreases as JNR increases with different gradient depending on the type of interference. Singletone continuous wave interference affects the gain of AGC the most, while band-limited white noise affects the gain of AGC the least. This tendency is correspondent to the results in [17]. Different types of interference affect the AGC gain differently and those effects can be characterized. From JNR 10dB, AGC Gain and JNR are linear in dB. Therefore, if the type of interference is known, it is possible to estimate JNR from AGC gain using this characterization results. The polynomial fitting results of the JNR versus AGC gain are in Table 1. The remaining problem of interference classification is solved by the algorithm using the adaptive lattice IIR notch filter parameters.

\subsection{Adaptive Lattice IIR Notch Filter Parameters for Interference Classification}

\subsubsection{Adaptive Lattice IIR Notch Filter}

Originally, the adaptive notch filter has been researched for continuous wave interference detection and mitigation

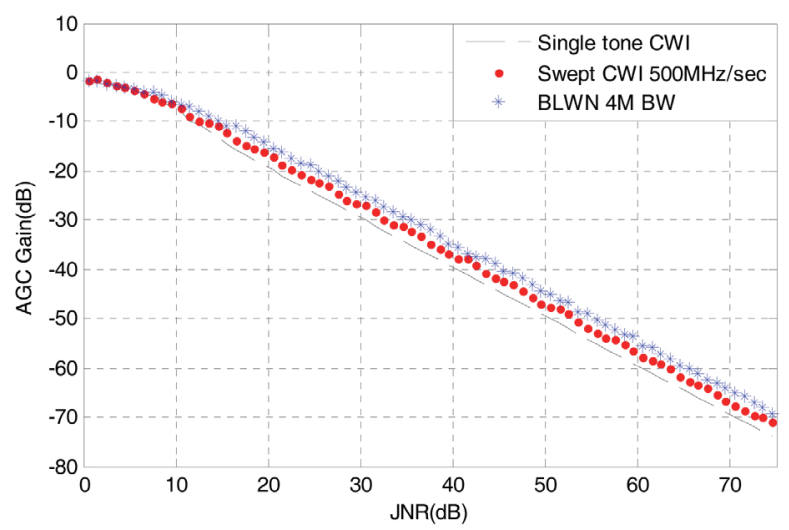

Fig. 1. AGC Characterization Results

Table 1. Polynomial Fitting Results from AGC characterizatio

\begin{tabular}{|c|c|}
\hline Type of Interference & $\begin{array}{c}\text { Polynomial Fitting Results for JNR vs AGC } \\
\mathrm{dB} \text { Gain (when JNR }>10)\end{array}$ \\
\hline $\begin{array}{c}\text { Single tone continuous wave } \\
\text { interference }\end{array}$ & $-1.014176 \mathrm{x}+1.175821$ \\
\hline $\begin{array}{c}\text { Swept continuous wave interference } \\
\text { (Sweep rate 500MHz/sec) }\end{array}$ & $-0.999496 \mathrm{x}+2.984180$ \\
\hline Band limited white Gaussian noise & $-0.990983 \mathrm{x}+4.879760$ \\
\hline
\end{tabular}


$[20,21]$. It is a low-complexity time domain approach which is computationally efficient compared to the frequency domain approach. In this paper, the adaptive lattice IIR notch filter structure is employed which was proposed in [22]. Adaptive lattice IIR notch filter parameters are used for the detection algorithm and the recursive least square (RLS) algorithm is used to estimate the continuous wave

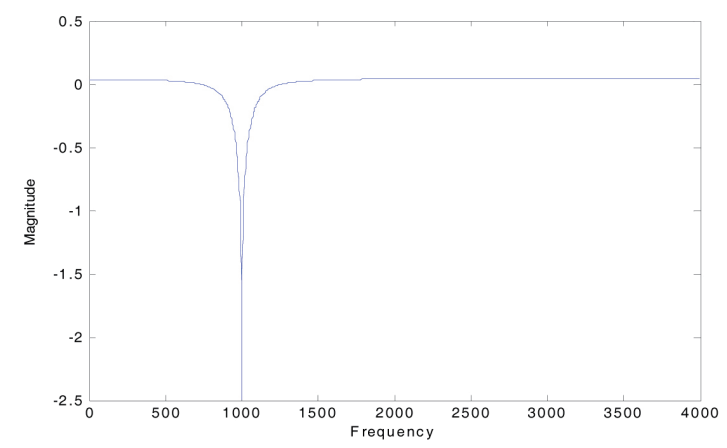

Fig 2. Magnitude Response of the Considered Notch Filter

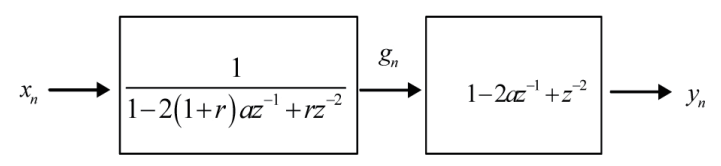

Fig. 3. Lattice IIR Filter Processing Block

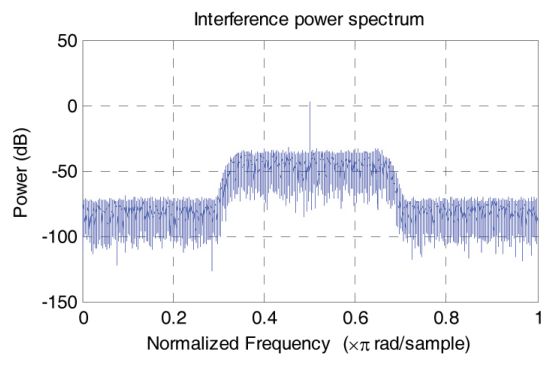

(a) Power Spectrum of CWI

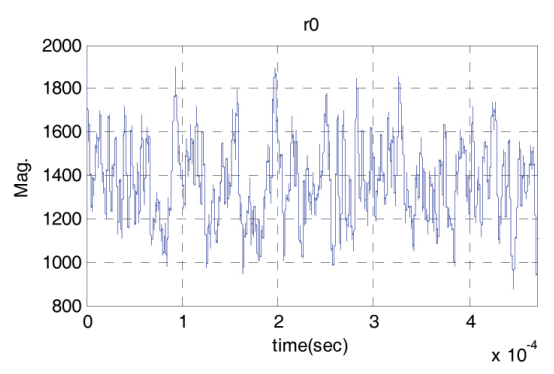

(c) $r_{0}$ interference frequency. This filter can be used for continuous wave interference excision. Fig. 2 presents the notch filter in the frequency domain. Single-tone continuous wave interference is represented as a spectral line in the frequency domain. The notch filter can eliminate signal components in that specific frequency region by placing a notch.

The transfer function of that notch filter can be represented as Eq. (5), where $\omega_{i}$ is the frequency of the interference signal, and $r$ is the distance between zero and pole. The lattice IIR filter can be viewed as a cascade of all-pole and all-zero lattice filters as shown in Fig. 3, where $a=\cos \left(\omega_{i}\right)$.

$$
H(z)=\frac{1-2 \cos \omega_{i} z^{-1}+z^{-2}}{1-2(1+r) \cos \omega_{i} z^{-1}+r z^{-2}}
$$

When the notch is placed at the interference signal's frequency, the output power is minimized. Therefore, the RLS algorithm uses the notch filter output power as a cost function $J$ in Eq. (6) and finds $a=\cos \left(\omega_{i}\right)$ which minimizes the cost function using Eq. (7) and (8).

$$
\begin{aligned}
& J=E\left[y_{n}^{2}\right] \\
& \frac{\partial J}{\partial a}=E\left[2 y_{n}\left(-2 g_{n-1}\right)\right]=E\left[2\left(g_{n}-2 a g_{n-1}+g_{n-2}\right)\left(-2 g_{n-1}\right)\right]=0 \\
& a=\frac{E\left[g_{n-1}\left(g_{n}+g_{n-2}\right)\right]}{2 E\left[g_{n-1}^{2}\right]}=\frac{r_{1}(n)}{r_{0}(n)}
\end{aligned}
$$

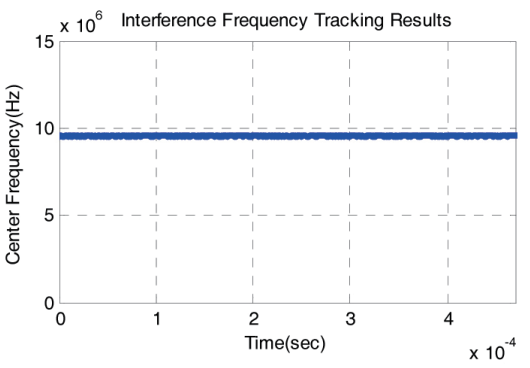

(b) Frequency Tracking Results

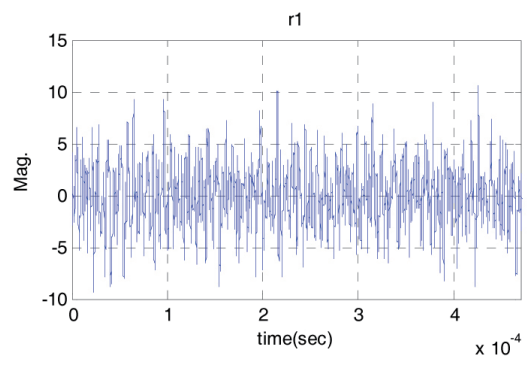

(d) $r_{1}$

Fig. 4. Power Spectrum and Filter Parameters of Continuous Wave Interference at L1 
Jeong Hwan Yang Intentional GNSS Interference Detection and Characterization Algorithm Using AGC and Adaptive IIR Notch...

$$
\omega=\arccos \left(\frac{r_{1}(n)}{r_{0}(n)}\right)
$$

where, $r_{1}(n)=\lambda r_{1}(n-1)+(1-\lambda)\left(g_{n-1}\left(g_{n}+g_{n-2}\right)\right)$, $r_{0}(n)=\lambda r_{0}(n-1)+(1-\lambda) 2 g_{n-1}^{2}$, and $\lambda$ is a forgetting factor for recursive calculation [23].

The frequency of continuous wave interference can be estimated using Eq. (9) with $r_{0}$ and $r_{1}$ from the recursive calculation. Fig. 4 demonstrates the power spectrum of a single-tone continuous wave interference and the adaptive notch filter's frequency tracking results, $r_{0}$, and $r_{1}$.

\subsubsection{Adaptive Lattice IIR Notch Filter Parameters for In- terference Detection and Classification}

The interference detection and classification algorithm utilizes $r_{0}$ and the standard deviation of the estimated frequency, $\sigma_{\hat{f}}$. Those parameters have unique characteristics according to the type of incoming interference signal. $r_{0}$ reflects the estimated power of the tracked signal by the filter, so it tends to converge to a certain value when CWI is present. Fig. 5. (a) shows the $r_{0}$ characteristic with and

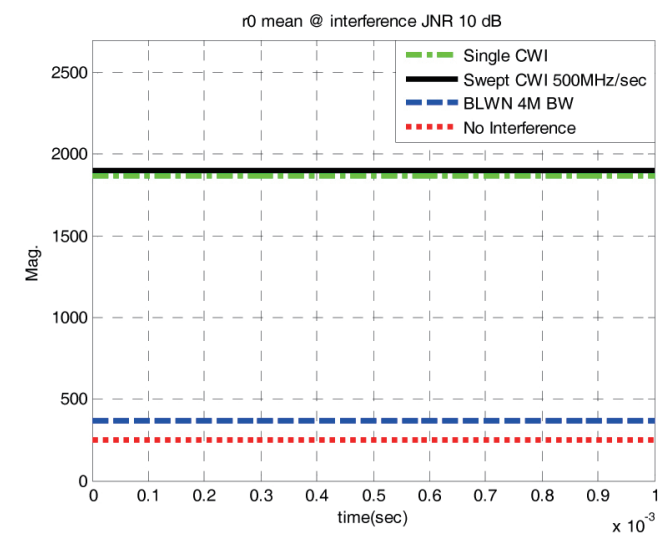

(a) Mean of $r_{0}$ without an interference signal. In addition, the standard deviation of the estimated frequency can be used to classify the type of interferences. $\sigma_{\hat{f}}$ of the single-tone continuous wave interference is comparably small because its frequency is fixed, while $\sigma_{\hat{f}}$ of swept continuous wave interference becomes larger according to the range that the frequency moves during $1 \mathrm{~ms}$ intervals. $\sigma_{\hat{f}}$ of the band-limited white noise is the largest because its frequency is random. Fig. 5. (b) shows the standard deviation of the frequency estimates for different interferences. In the proposed algorithm, the mean of $r_{0}$ for $1 \mathrm{~ms}$ and the estimated frequency's standard deviation for $1 \mathrm{~ms}$ were used as test statistics.

\section{Interference Detection and Characteriza- tion Algorithm}

In this section, the interference detection and characterization algorithm is proposed by using AGC gain, adaptive lattice IIR notch filter parameters, and AGC characterization data. The parameter, $r_{0}$ from the adaptive notch filter is useful to detect continuous wave

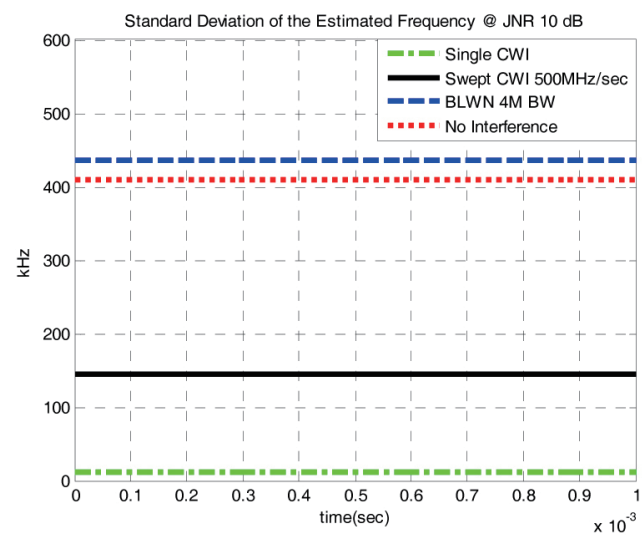

(b) $\sigma_{\hat{f}}$

Fig. 5. The characteristics of $r_{0}$ and $\sigma_{\hat{f}}$ according to different types of interference

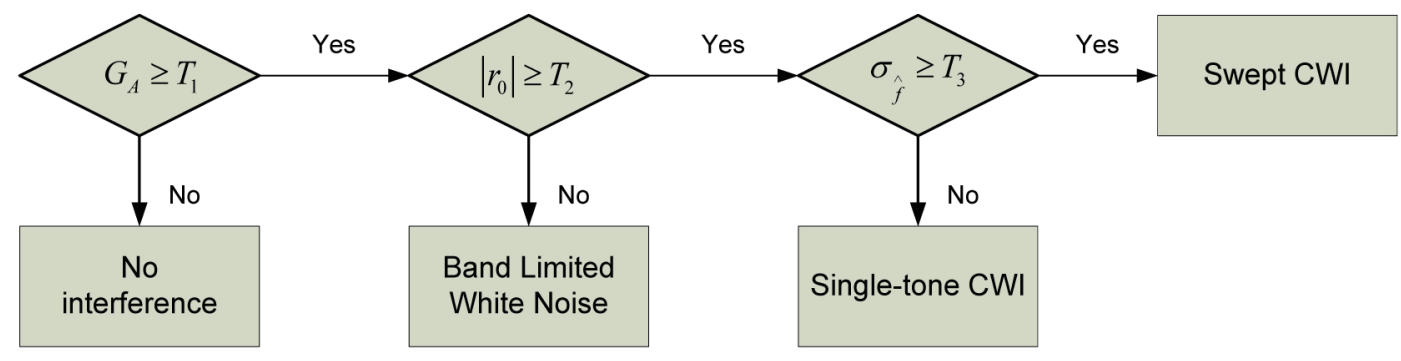

Fig. 6 Interference Detection and Classification Algorithm 


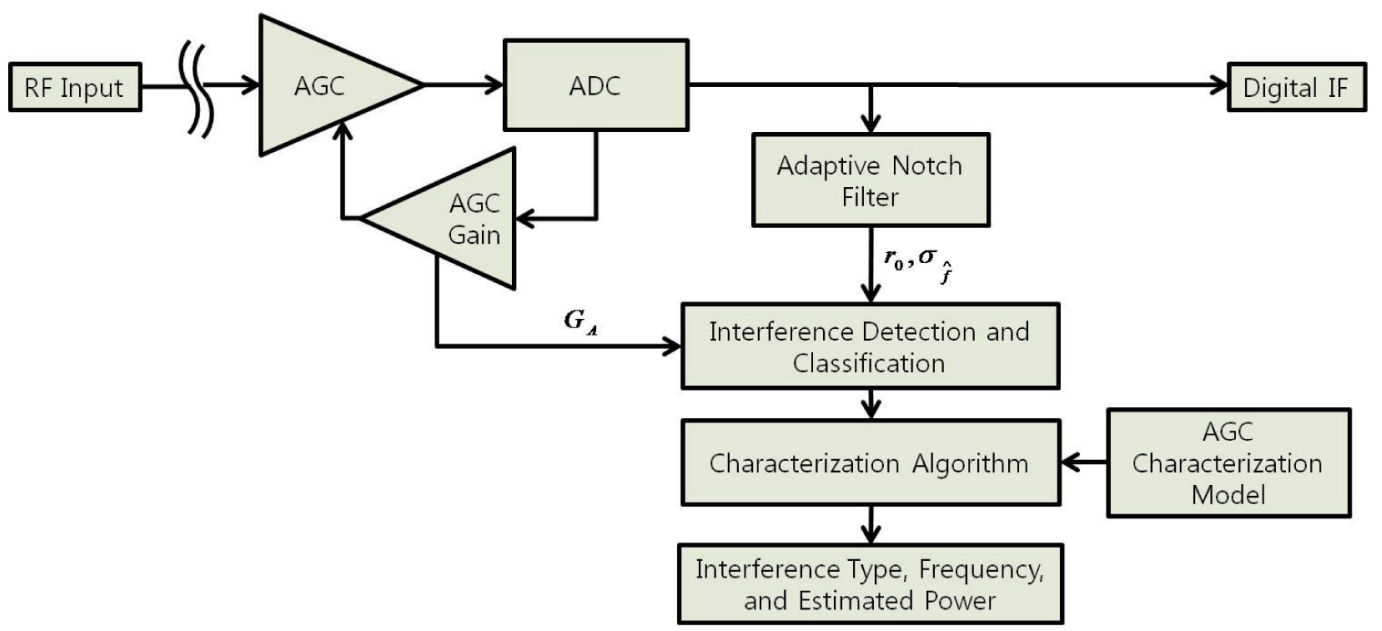

Fig. 7 Interference Detection and Characterization Algorithm in a GNSS receiver

interference. However, $r_{0}$ does not reflect the power of the band limited white noise, therefore it is difficult to set a detection threshold of band limited white noise using $r_{0}$ . However, the AGC gain is sensitive to all interference types. Therefore, the proposed algorithm uses AGC gain as interference detection parameter. The proposed detection and classification algorithm is explained in Fig. 6. First, the AGC gain is compared to a threshold to detect the presence of an interference signal. Second, the algorithm classifies CWI and band limited white noise by the mean of $r_{0}$ magnitude. Third, the algorithm classifies the singletone CWI and swept CWI using the standard deviation of the estimated frequency. Furthermore, the power of interference is estimated with the measured AGC gain based on the AGC gain characterization data. The proposed algorithm enables power estimation of three types of interferences including

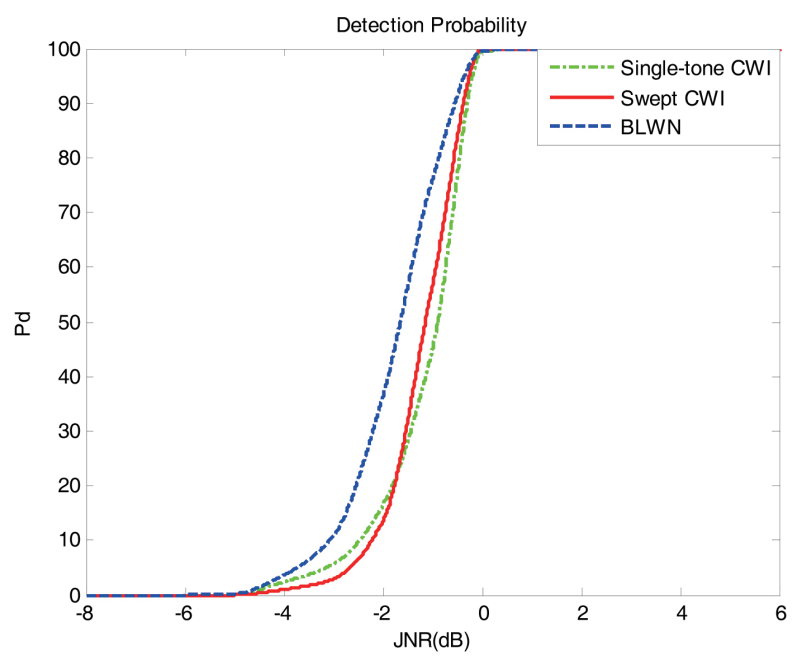

Fig 8. Detection Probability of the Interference Signals single-tone continuous wave interference, swept continuous wave interference, and band-limited white noise. Using this algorithm, more accurate power estimation is possible and this can lead to more accurate interference localization. Fig. 7 presents the flow chart of the overall algorithm in a reference GNSS receiver.

\section{Performance Evaluation of the Proposed Algorithm}

The selection of the detection threshold has a great effect on the performance of the interference detection. In this paper, the threshold is selected to detect the interference signal whose JNR is more than $0 \mathrm{~dB}$, which affects the accuracy of GNSS receivers. To obtain the optimum detection parameters, the false alarm rate is fixed to 0.01 , based on the Neyman-Pearson approach. To evaluate the proposed algorithm's performance, continuous wave interferences, swept continuous wave interferences, and band-limited white noise signals are generated with power levels from JNR $-15 \mathrm{~dB}$ to JNR 35dB. The detection probability has been calculated by Monte Carlo simulations, on the basis of 300 independent runs of the detection algorithm. Fig. 8 refers to the detection probability of the single-tone continuous wave interference, swept continuous wave interference, and band limited white noise. In addition, the power estimation performance was evaluated by comparing true interference power and estimated interference power by the characterization algorithm. The mean of the estimated interference power and its RMS errors according to different power levels are outlined in Table 2. The results show that the algorithm can estimate interference power with less than $1 \mathrm{~dB}$ RMS error. 
Jeong Hwan Yang Intentional GNSS Interference Detection and Characterization Algorithm Using AGC and Adaptive IIR Notch...

Table 2. Estimated JNR and RMS Errors

\begin{tabular}{|c|c|c|c|c|c|c|}
\hline \multirow{2}{*}{$\begin{array}{c}\text { True JNR } \\
(\mathrm{dB})\end{array}$} & \multicolumn{2}{|c|}{ Single-tone CWI } & \multicolumn{2}{c|}{ Swept CWI } & \multicolumn{2}{c|}{ Band Limited White Noise } \\
\cline { 2 - 7 } & $\begin{array}{c}\text { Mean of } \\
\text { Estimated } \\
\text { JNR (dB) }\end{array}$ & $\begin{array}{c}\text { RMS Error } \\
(\mathrm{dB})\end{array}$ & $\begin{array}{c}\text { Mean of } \\
\text { Estimated } \\
\text { JNR }(\mathrm{dB})\end{array}$ & $\begin{array}{c}\text { RMS Error } \\
(\mathrm{dB})\end{array}$ & $\begin{array}{c}\text { Mean of } \\
\text { Estimated } \\
\text { JNR }(\mathrm{dB})\end{array}$ & $\begin{array}{c}\text { RMS Error } \\
(\mathrm{dB})\end{array}$ \\
\hline 10 & 9.0155 & 0.9898 & 10.3895 & 0.3985 & 11.4081 & 1.4162 \\
\hline 15 & 15.4256 & 0.4278 & 15.1898 & 0.2210 & 15.6662 & 0.7267 \\
\hline 20 & 20.8033 & 0.8044 & 20.2571 & 0.2618 & 20.4131 & 0.4540 \\
\hline 25 & 25.8378 & 0.8382 & 25.1535 & 0.1629 & 25.4529 & 0.5255 \\
\hline 30 & 30.7946 & 0.7947 & 30.0507 & 0.0623 & 30.4366 & 0.4584 \\
\hline 35 & 35.7327 & 0.7328 & 35.0318 & 0.0373 & 35.3157 & 0.3910 \\
\hline
\end{tabular}

\section{Conclusion}

In this paper, the interference detection and characterization algorithm was proposed using AGC and adaptive lattice IIR notch filter parameters. AGC characterization results showed how the AGC of a reference receiver responds differently to different types of RFI. A linear model was found to be appropriate for modeling the AGC response against interference with JNR above 10 dB. Adaptive notch filter parameters were examined for interference detection and classification purpose. As a result, the mean of and the standard deviation of estimated frequency, were found to be suitable for detection and classification of single-tone continuous wave interference and swept continuous wave interference. Furthermore, in order to detect band limited white noise, the interference detection and characterization algorithm was designed by combining AGC and adaptive notch filter parameters. The simulation results demonstrated that the proposed interference detection and characterization algorithm can successfully detect and characterize three different interferences. The algorithm can detect interferences above JNR $0 \mathrm{~dB}$ with $100 \%$ detection probability. This algorithm can be used for intentional GNSS interference monitoring and enhancement of the interference mitigation algorithm.

\section{Acknowledgement}

This work has been supported by National GNSS Research Center program of Defense Acquisition Program Administration and Agency for Defense Development.

\section{References}

[1] Pullen, S., Gao, G., Tedeschi, C., and Warburton, J., "The Impact of Uninformed RF Interference on GBAS and
Potential Mitigations", Proceedings of the 2012 ITM ION, Newport Beach, CA, 2012.

[2] Luo, M., Xie, G., Akos, D., and Pullen, S., "Radio Frequency Interference Validation Testing for LAAS using the Stanford Integrity Monitor Testbed", Proceedings of ION NTM 2003, Anaheim, CA, 2003.

[3] Joo, I.W., Sin, C.S., Kim, J.H., and Lee, J.H., "Technical Trends of Monitoring GPS Jamming”, Electronics and Telecommunications Trends, Vol. 26, No. 4, 2011, pp.115-122.

[4] Brown, A., Atterberg, S., and Gerein, N., "Detection and Location of GPS Interference Sources Using Digital Receiver Electronics", Proceedings of ION Annual Meeting, San Diego, CA, 2000.

[5] Brown, A., Reynolds, D., Roberts, D., Serie, S., "Jammer and Interference Location System - Design and Initial Test Results", Proceedings of the ION GPS, Nashville, TN, 1999.

[6] Ward, P.W., "What's Going On?: RFI Situational Awareness in GNSS Receivers", InsideGNSS, Vol. 2, No. 6, 2007, pp 34-42.

[7] Ward, P.W., "Simple Techniques for RFI Situational Awareness and Characterization in GNSS Receivers", Proceedings of ION National Technical Meeting, San Diego, CA, 2008.

[8] Ndili, A., and Enge, P., "GPS Receiver Autonomous Interference Detection", Proceeding of Position Location and Navigation Symposium, Palm Springs, CA, 1998.

[9] Yun, Y.S., Kee, C.D., Rife, J., Luo, M., Pullen., S. and Enge., P, "Detecting RFI Through Integrity Monitoring at a DGPS Reference Station", Proceedings of the 61st Annual Meeting of the ION, Cambridge, MA, 2005.

[10] Balaei, A.T., Dempster, A.G., and Barnes, J., "A Novel Approach in Detection and Characterization of CW Interference of GPS Signal Using Receiver Estimation of C/No", Proceedings of Position, Location, And Navigation Symposium 2006, San Diego, CA, 2006.

[11] Chen, D., Yang, T., Jin, J., Mao, C., and Zhou, J, “A trimode Compass/GPS/Galileo RF receiver with all-digital 
automatic gain control loop", Analog Integr Cir Sig Process, Vol. 70, 2012, pp. 69-77. DOI: 10.1007/s10470-011-9656-Z

[12] Kim, N., "Interference Effects on GPS Receivers in Weak Signal Environments”, Master's Thesis, Department of Geomatics Engineering, University of Calgary, Calgary, Alberta, Canada, 2006.

[13] Yang, J.H., Kang, C.H., Kim, S.Y., and Park, C.G, “The Design of the GPS L1 Software Simulator using MATLAB SIMULINK", Proceedings of the $18^{\text {th }}$ GNSS Workshop, Jeju, Korea, 2011.

[14] Wildemeersch, M., Pons, E.C., Rabbachin, A., and Guasch, J.F., "Impact Study of Unintentional Interference on GNSS Receivers", EC Joint Research Centre Scientific and Technical Reports, Institute for the Protection and Security of the Citizen, European Union, 2010.

[15] Thompson, R., Cetin, E., and Dempster, A., "Detection and Jammer-to-Noise Ratio Estimation of Interferers Using the Automatic Gain Control," Proceedings of IGNSS Symposium, Sydney, Australia, 2011.

[16] Batistide, F., Akos, D.M., Macabiau, C., and Roturier, B., "Automatic Gain Control(AGC) as an Interference Assessment Tool", Proceedings of ION GNSS, Portland, OR, 2003.

[17] Isoz, O., Akos, D., and Lindgren, T., "Assessment of GPS L1/Galileo E1 Interference Monitoring System for the
Airport Environment", Proceedings of the ITM ION GNSS 2011, Portland, OR, 2010.

[18] Isoz, O., Balaei, A.T., and Akos, D., "Interference Detection and Localization in GPS L1 Band", Proceedings of the ITM ION 2010, San Diego, CA, 2010.

[19] Kaplan, E. D., and Hegarty, C. J. “Understanding GPS: Principles and Applications", Artech House, Norwood, MA, 2006.

[20] Borio, D., Camoriano, L, and Presti, L., "Two-Pole and Multi-Pole Notch Filters: a Computationally Effective Solution for Interference Detection and Mitigation", IEEE Systems Journal, Vol. 2, No.1, Issue 1, 2008, pp. 38-47. DOI: 10.1109/JSYST.2007.914780

[21] Chien, Y., Huang, Y., Yang, D, and Tsao, H., "A Novel Continuous Wave Interference Detectable Adaptive Notch Filter for GPS Receivers", Proceedings of 2010 IEEE Global Telecommunications Conference, Miami, FL, 2010.

[22] Kim, S. Y., Kang, C.H., Yang, J.H., and Park, C.G., "GNSS Interference Detection using Adaptive Notch Filter", Proceedings of The Korean Society for Aeronautical \& Space Science Conference, Gangwondo, South Korea, 2012.

[23] Cho, N. I., and Lee, S. U., "On the Adaptive Lattice Notch Filter for the Detection of Sinusoids", IEEE Transactions on Circuits and Systems - II: Analog and Digital Signal Processing, Vol. 40, No. 7, 1993, pp. 405-416. 